\title{
A novel model to label delirium in an intensive care unit from clinician actions
}

\author{
Caitlin E. Coombes ${ }^{1}$, Kevin R. Coombes ${ }^{2}$ and Naleef Fareed ${ }^{2,3^{*}}$ (1)
}

\begin{abstract}
Background: In the intensive care unit (ICU), delirium is a common, acute, confusional state associated with high risk for short- and long-term morbidity and mortality. Machine learning (ML) has promise to address research priorities and improve delirium outcomes. However, due to clinical and billing conventions, delirium is often inconsistently or incompletely labeled in electronic health record (EHR) datasets. Here, we identify clinical actions abstracted from clinical guidelines in electronic health records (EHR) data that indicate risk of delirium among intensive care unit (ICU) patients. We develop a novel prediction model to label patients with delirium based on a large data set and assess model performance.
\end{abstract}

Methods: EHR data on 48,451 admissions from 2001 to 2012, available through Medical Information Mart for Intensive Care-III database (MIMIC-III), was used to identify features to develop our prediction models. Five binary ML classification models (Logistic Regression; Classification and Regression Trees; Random Forests; Naïve Bayes; and Support Vector Machines) were fit and ranked by Area Under the Curve (AUC) scores. We compared our best model with two models previously proposed in the literature for goodness of fit, precision, and through biological validation.

Results: Our best performing model with threshold reclassification for predicting delirium was based on a multiple logistic regression using the 31 clinical actions (AUC 0.83). Our model out performed other proposed models by biological validation on clinically meaningful, delirium-associated outcomes.

Conclusions: Hurdles in identifying accurate labels in large-scale datasets limit clinical applications of ML in delirium. We developed a novel labeling model for delirium in the ICU using a large, public data set. By using guidelinedirected clinical actions independent from risk factors, treatments, and outcomes as model predictors, our classifier could be used as a delirium label for future clinically targeted models.

Keywords: Delirium, Electronic health records, Intensive care unit, Predictive model, Risk factors

\section{Background}

Delirium is an acute, confusional state associated with a fluctuating disturbance in awareness and cognition arising alongside serious illness [1]. In the intensive care unit (ICU), delirium affects up to $41-50 \%$ of patients overall $[2,3]$, up to $82 \%$ of patients with prolonged ICU length

*Correspondence: naleef.fareed@osumc.edu

${ }^{2}$ Department of Biomedical Informatics, The Ohio State University

College of Medicine, 460 Medical Center Dr., 512 Institute of Behavioral

Medicine Research, Columbus, OH 43210, USA

Full list of author information is available at the end of the article of stay (LOS) [3], and over $75 \%$ of patients undergoing mechanical ventilation [4]. Patients with in-hospital delirium are at risk for adverse short- and long-term outcomes, including increased LOS, discharge to postacute nursing facilities [3, 5-7], slowed surgical recovery [8], persistent cognitive impairment [9], incident dementia [10], and death [10].

Delirium poses challenges for both researchers and clinicians from incompletely understood pathophysiology $[3,5]$, multifactorial etiology $[3,11]$, terminological inconsistency [5], and under-recognition and inappropriate management in the clinical setting $[3,5]$. The clinical 
presentation of the syndrome is broad, including an agitated, hyperactive subtype; a somnolent, hypoactive subtype; or mixed features [5]. The hypoactive subtype is less frequently diagnosed and has poorer prognosis [5]. Additional patients may manifest with subsyndromal delirium or "attenuated delirium syndrome": a subclinical confusional state meeting part, but not all, of the DSM-5 criteria for delirium [12]. Due in part to delirium's comorbid presentation with serious illness, advanced age, depression, and dementia[5, 12] and its heterogeneous and fluctuating symptom presentation [12], delirium is often under-recognized in the hospital [5, 12, 13]. Because delirium arises comorbidly, the primary treatment is identification, diagnosis, and treatment of the etiologic organic illness or toxic insult, accompanied by pharmacological and nonpharmacological delirium symptom management [11]. These challenges make delirium an important target of machine learning (ML) [14-22].

Training ML models require a valid delirium label which can accurately capture a patient with the condition. For a method of labeling to be useful as a foundation for clinical prediction, it must be independent of both risk factors and outcomes of interest. Although the gold standard is a provider-administered screening tool such as the Confusion Assessment Method for the ICU (CAM-ICU) [13, 23], these labor-intensive identifiers must be prospectively administered and are not available in all settings [13, 20-22], revealing a need for a delirium identifier that can be abstracted retrospectively and computationally from the medical record.

Two preliminary studies on small cohorts $(<400$ patients) have proposed other simple, chart-based labels when CAM-ICU is absent. Kim et al.[24] used the CAMICU and provider interview as the gold standard to label delirium with modest sensitivity (30\%), high specificity $(97 \%)$ and high positive predictive value $(\mathrm{PPV}=83 \%)$ from the presence of either an International Classification of Diseases (ICD) code or antipsychotics use, with improved sensitivity for delirium that was hyperactive or mixed type (64\%) or severe (73\%). By chart review, Puelle et al.[25] identified eight key words or phrases (altered mental status, delirium, disoriented, hallucination, confusion, reorient, disorient and encephalopathy) with high PPV (60-100\%) for delirium (model sensitivity and specificity not reported).

Here we present an assessment of three methods to label delirium in the chart from medical record events. We propose a supervised binary classifier based on counts of 31 clinician actions, including medications, orders, and clinical impressions in free-text notes. All 31 predictors are independent of risk factors and outcomes of interest, generating a labeling method that could be used as a foundation for downstream clinical predictions.
We compare this model to Kim et al's classification based on ICD code and antipsychotics use ("Kim's classifier") and to Puelle et al's eight words with high PPV ("Puelle's classifier"). To the best of our knowledge, we are the first to test these proposals on a large-scale dataset. Because our dataset is too large to permit chart review and CAMICU is unavailable, we set ICD code as our initial delirium identifier. We assess the quality of classification of each model by biological validation[26] on clinically meaningful, delirium-associated outcomes, demonstrating superior performance with our model of 31 clinician actions. Our model has the potential to be generalized and implemented across ICU datasets to support improved labeling for downstream clinical predictive modeling.

\section{Strategies to label and validate delirium in large-scale datasets}

In 2015, Inouye et al. proposed research priorities for delirium, including improved diagnosis and subtyping, stratification of high risk patients, biomarker detection, and identification of genetic determinants [3]. Researchers have since applied unsupervised ML, including clustering[15] and latent class analysis [14], to subtype patients. More commonly, supervised ML is used to predict delirium incidence within an ICU stay based on a priori risk factors [21], heart rate variability [17], or medical record events from the first $24 \mathrm{~h}$ of hospitalization $[16,18,20,27]$.

To make clinically actionable predictions, the researcher requires a delirium label that is independent of the clinical covariates and predictors of interest. The preferred measures in clinical practice for labeling delirium are nurse- or provider-administered, validated screening tools, including the CAM-ICU[13, 23] and the Intensive Care Delirium Screening Checklist (ICDSC) $[13,28,29]$. CAM-ICU administered during treatment is a mainstay label of delirium in the ML research setting [14-19]. However, variations in institutional practice and physician buy-in can lead to inconsistent use of the CAM or ICDSC in the clinical setting [13]. When CAM-ICU is unavailable or suspect, researchers may employ nurse chart review [20, 21]. However, chart review relies on clinical judgment[25] and poses time and labor costs that grow prohibitive as data sets increase in size.

Other researchers have used ICD codes as a delirium label [22]. Though convenient, ICD codes, especially secondary codes (such as delirium in a critical illness setting), are prone to high levels of missingness and inaccuracy [30-32]. Although the prevalence of delirium in the ICU has been estimated to be as high as $24-82 \%$ [2-4], published models have been built using ICD code labels for delirium that may be as sparse as 3.1\% [22]. This mismatch between proportion of expected patients 
with delirium and available ICD codes suggests a risk of outcome misclassification if ICD codes are used, with potential for serious bias in learned model outputs [33]. Weaknesses in delirium labeling underlying much stateof-the-art research calls the generalizability and clinical utility of these studies into question.

Various tools are available when binary outcome misclassification in a dataset is suspected. Sensitivity analysis can be used to adjust the summary output of a logistic regression model, but it relies heavily on frequency estimates supplied by the researcher's a priori knowledge of the field, and cannot be learned from the model [33]. For some binary classifiers, outcome misclassification can be addressed by tuning model cut-points based on a priori knowledge or researcher goals for sensitivity or specificity or properties of the receiver operating curve (ROC) to enact a desired reclassification, a core practice in diagnostic test development[34] with applications in supervised model refinement [16].

Assessing outcome reclassification on real data is challenging due to absence of a gold standard. However, the concern is pressing: unless model fit is perfect (sensitivity and specificity $=100 \%$ ), all binary classification inherently generates some degree of "outcome reclassification," where members labeled as belonging to one group when entering the model are later predicted to belong to the other group. For clinical regression models, Harrell et al. proposed that the concordance index or c-index, calculated from pairwise comparisons of a prognostic indicator between classified and reclassified subjects, could be employed as a "clinically meaningful" measure of model goodness-of-fit [37]. We have previously proposed the related principle of biological validation: that ML assignments can be meaningfully validated by employing wellunderstood biological outcomes when ground-truth is unavailable [26]. Inspired by Harrell's approach, we compare five prognostic measures between classified and reclassified groups to biologically validate outcome reclassification and model goodness-of-fit for delirium identification.

\section{Methods}

\section{Study population}

Study data were drawn from Medical Information Mart for Intensive Care-III (MIMIC-III), a freely available database of electronic health record (EHR) data collected on 63,157 intensive care unit (ICU) admissions at Beth Israel Deaconess Medical Center from 2001 to 2012 [38-41]. Delirium within a hospitalization was defined by ICD-9 code [24]. (Additional file 2: Table A.1) Unique admissions were included for all adult patients $\geq 18$ years of age with ICU length-ofstay (LOS) less than 31 days (48,451 hospitalizations).
Restricting LOS removed 2,315 outlier hospitalizations (4.6\%) with LOS up to 295 days. From the cohort population, $25 \%$ of positives and negatives were randomly sampled and reserved for a test set (12,135 admissions), retaining $75 \%$ for training $(36,406$ admissions).

\section{A novel model predicting delirium from clinician actions Variable selection}

We proposed a model to label presence of delirium in a chart based on clinician actions. We hypothesized that changes in clinical actions concordant with diagnostic work-up for delirium can serve as an indicator that the clinical team had made a delirium diagnosis. Clinician actions presumed to indicate a response to delirium onset were identified from published guidelines for delirium work-up and abstracted from electronic health record (EHR) data. These included 18 laboratory and imaging orders and 4 medications [13, 42]. Pharmacologic interventions were selected based on evidence of widespread use for the management of delirium, not by efficacy or other clinical measures [13]. Clinical impressions were extracted from the presence of eight words or phrases with high PPV for delirium in EHR notes [25]. Additional file 2: Table A.2 lists the 31 included clinical actions. No steps were taken to identify or impute missing values. Occurrence of clinician actions were formed into an event count matrix across each admission [43]. A more detailed description of data pre-processing, with code, is available in Additional file 1: File B.

\section{Supervised model selection and refinement}

We compared performance of five binary ML classifiers $[16,17,19,22]$, including logistic regression (stats R-package), Classification and Regression Trees (CART; rpart R-package) [44, 45], supervised random forests (randomForest) [46, 47], naïve Bayes (e1071) [48, 49], and support vector machines (SVM; e1071) [49, 50]. (Additional file 1: File A.1) The logistic regression model underwent refinement and feature selection by stepwise forwards and backwards selection, L1/LASSO (Least Absolute Shrinkage and Selection Operator) penalization [51, 52], L2/Ridge penalization [53], and combined L1-L2 penalization (penalized). [54] Model performance on the training set was compared by ROC visualization and AUC (pROC) [55]. (Additional file 1: File A.2) The top performing model was selected by maximum AUC. Model development is reported here in accordance with Transparent Reporting of a multivariable prediction model for Individual Prognosis or Diagnosis (TRIPOD) guidelines [56]. 


\section{Reclassification and binary threshold determination}

Logistic regression generates a model with a log-odds threshold set at zero to divide hospitalizations with incident delirium from those without. This "natural" or "default" cut-point reflects the prior probability of delirium within the cohort, and is therefore susceptible to error from outdated prior information (such as known misclassification). As commonly implemented in diagnostic test development, we tuned the cut-point of our binary classifier to calibrate sensitivity and specificity to correct for known misclassification [34], a technique in practice in delirium supervised model development [16]. Because we suspect ICD-9 code missingness [30-32], we desire a model with high sensitivity. In the case of known misclassification, we believe that some of the additional positives generated by increased sensitivity represent true, but unlabeled, positives that have been reclassified. These reclassified positives represent hospitalizations containing real incident delirium, but lacking ICD-9 codes due to a priori outcome misclassification from known ICD-9 code missingness [30-32]. Thus, reclassification by up-tuning sensitivity allows us to generate a model that better labels the presence of true delirium.

On training data, we compared six algorithmic methods for reclassification of a binary model by tuning sensitivity: the Youden index [57], maximizing both sensitivity and specificity, maximizing accuracy, minimizing the distance to $\operatorname{ROC}(0,1)$, maximizing accuracy given a minimum constraint of sensitivity, and maximizing sensitivity given a minimal specificity constraint (Additional file 1: A.3; cutpointr R-package) [58]. We determined the threshold of choice based on concordance between measures, choosing a cut-point that represented trends between tuning methods. We also visualized reclassification by each cut-point by density plot.

The final model was trained on training data using the binary classifier with highest AUC, selected by maximum AUC, and the cut-point with highest measured concordance. This best-performing model was run on retained test data. Validation was performed on test data only.

\section{Comparison models}

We identified two related models in the literature proposed from chart review to predict incidence of delirium within a hospital stay from clinician actions and implemented them at an expanded scale.

To assess Puelle's classifier [25], we trained a logistic regression model with eight binary predictors for presence or absence at any point in a hospitalization of eight words in notes with high PPV for delirium on the training set (Additional file 1: Material A.4.1). Previously, we had implemented the same eight words in our model of
31 clinician actions (Additional file 2: A.2). We omitted Puelle's final criterion, “'alert and oriented' $(<3)$ ” due to difficulty of abstracting this data point from free-text note fields without natural language processing. The resultant model was validated on the test set. The binary threshold was chosen with the Youden Index. We compared our novel model to Puelle's classifier by the Akaike Information Criterion (AIC) and the Bayes Information Criterion (BIC) [59].

We tested Kim's classifier [24] by labeling hospitalizations as delirium-positive if they contained a delirium ICD-9 code or if anti-psychotics were prescribed at any point during hospitalization (Additional file 1: Material A.4.2). Admissions were delirium-negative if a delirium ICD-9 code was not applied and anti-psychotics were not administered. This simple recategorization did not require training and was applied directly to the test set.

\section{Validation of reclassified models by clinical markers and outcomes}

Statistical measures of final model performance included sensitivity, specificity, PPV, negative predictive value (NPV), AUC (for supervised models), and comparison against expected prevalence of ICU delirium.

Reclassification was validated on five clinically meaningful demographic and outcome measures: age at admission [3], discharge location [5-7], death in hospital, death within 30 days of admission [38], and one-year mortality from admission [10]. To assess success and meaningfulness of re-classification and goodness-of-fit for each model, we separated admissions into four groups (Table 1). First, we compared ICD-Positives and Double-Negatives. If these were significantly different, we report tests comparing ICDPositives to Reclassified-Positives, Double-Negatives to Reclassified-Negatives, and Reclassified-Positives to Reclassified-Negatives. Similarity or difference between groups was assessed using Tukey multiple comparisons

Table 1 Definitions of four classified and re-classified categories generated by a binary classifier

\begin{tabular}{lll}
\hline Classification group & $\begin{array}{l}\text { Has delirium ICD-9 } \\
\text { code }\end{array}$ & $\begin{array}{l}\text { Model } \\
\text { predicts } \\
\text { delirium }\end{array}$ \\
\hline Double-Positives & Yes & Yes \\
Reclassified-Positives & No & Yes \\
Reclassified-Negatives & Yes & No \\
Double-Negatives & No & No
\end{tabular}

For any binary classifier with less than $100 \%$ accuracy, model testing results in some degree of reclassification of positives and/or negatives, generating four groups. For example, some admissions with an ICD-9 code for delirium are labeled as negative by the model, leading to re-classification 
Table 2 Demographic characteristics of a cohort of adult ICU patients

\begin{tabular}{|c|c|c|c|c|}
\hline & $\begin{array}{l}\text { Total } \\
\mathrm{n}(\%)^{\mathrm{a}}\end{array}$ & $\begin{array}{l}\text { Patients with Delirium ICD-9 } \\
\text { Code } \\
\text { n }(\%)^{\mathrm{b}}\end{array}$ & $\begin{array}{l}\text { Patients without Delirium ICD-9 } \\
\text { Code } \\
\mathrm{n}(\%)^{\mathrm{b}}\end{array}$ & p-Value \\
\hline Total & 48,541 & 3,850 & 44,691 & - \\
\hline \multicolumn{5}{|l|}{ Sex } \\
\hline Male & $27,220(56.1 \%)$ & $2,181(56.6 \%)$ & $25,039(56.0 \%)$ & \multirow[t]{2}{*}{$0.538^{c}$} \\
\hline Female & 21,321 (43.9\%) & $1,675(43.4 \%)$ & 19,646 (44.0\%) & \\
\hline \multicolumn{5}{|l|}{ Race/Ethnicity } \\
\hline White or Caucasian & 34,792 (71.7\%) & 2,857 (74.1\%) & 31,935 (71.5\%) & \multirow[t]{5}{*}{$<0.005^{d}$} \\
\hline Black or African & 4,668 (9.6\%) & 381 (9.9\%) & 4,287 (9.6\%) & \\
\hline Hispanic or Latino & $1,720(3.5 \%)$ & $129(3.4 \%)$ & $1,591(3.6 \%)$ & \\
\hline Asian & $1,133(2.3 \%)$ & $74(1.9 \%)$ & $1,059(2.4 \%)$ & \\
\hline Other $^{e}$ & $6,228(12.8 \%)$ & $415(10.8 \%)$ & $5,813(13.0 \%)$ & \\
\hline \multicolumn{5}{|l|}{ Age at Admission ${ }^{7}$} \\
\hline$<30$ years & $2,222(4.6 \%)$ & $140(3.6 \%)$ & $2,082(4.6 \%)$ & \multirow[t]{8}{*}{$<0.0005$} \\
\hline 30-39 years & $2,568(5.3 \%)$ & $164(4.3 \%)$ & $2,404(5.4 \%)$ & \\
\hline 40-49 years & $5,151(10.6 \%)$ & $388(10.1 \%)$ & $4,763(10.7 \%)$ & \\
\hline $50-59$ years & $8,396(17.3 \%)$ & $607(15.7 \%)$ & 7,789 (17.4\%) & \\
\hline 60-69 years & $10,117(20.8 \%)$ & $715(18.5 \%)$ & $9,402(21.0 \%)$ & \\
\hline 70-79 years & $10,042(20.7 \%)$ & $789(20.5 \%)$ & $9,253(20.7 \%)$ & \\
\hline 80-89 years & 7,432 (15.3\%) & 747 (19.4\%) & $6,685(15.0 \%)$ & \\
\hline$\geq 90$ years & $2,613(5.4 \%)$ & $306(7.9 \%)$ & $2,307(5.2 \%)$ & \\
\hline \multicolumn{5}{|l|}{ Length of Stay ${ }^{g}$} \\
\hline$<5$ days & 17,406 (35.9\%) & 783 (20.3\%) & $16,623(37.2 \%)$ & \multirow[t]{4}{*}{$<0.0005$} \\
\hline 5 - 10 days & 19,131 (39.4\%) & $1,470(38.1 \%)$ & $17,661(39.5 \%)$ & \\
\hline $11-20$ days & 9,163 (18.9\%) & 1,149 (29.8\%) & 8,014 (17.9\%) & \\
\hline $21-30$ days & $2,841(5.9 \%)$ & $454(11.8 \%)$ & 2,387 (5.3\%) & \\
\hline
\end{tabular}

Differences between patients with and without delirium ICD-9 codes were tested with t-tests and chi-squared tests, as appropriate

a Percent of total patients

b Percent within subgroup (with or without delirium ICD-9 code)

c Pearson's Chi-squared test with Yates' continuity correction

d Pearson's Chi-squared test

e Other race, race unavailable, multi race ethnicity ( $0.21 \%$ of total cohort), Native American, Native Hawaiian, or Pacific Islander ( $0.06 \%$ of cohort)

f Welch's two-sample t-test

g Continuous variable in the MIMIC-III discretized for illustration in this table

of means for continuous data and Pearson's chi-squared tests with pairwise comparisons with the Holm correction for categorical data $[60,61]$.

\section{Results}

From 48,451 unique adult admissions in MIMIC-III with $\operatorname{LOS} \leq 31$ days, we identified 3,850 patients with delirium by ICD-9 codes (7.9\%). Demographic characteristics and pertinent outcomes of the cohort are described in Table 2. Briefly, the group with patients with delirium had statistically significant differences with the group without delirium for race/ethnicity, age at admission, and length of stay.

\section{Novel model of 31 clinician actions}

Figure 1 summarizes the performance of five supervised binary classifiers by ROC. Logistic regression performed best on the training set $(A U C=0.83)$. Naïve Bayes, SVM, CART, and random forests produced models with AUC of 0.66, 0.61, 0.59, and 0.58, respectively. Attempts to refine the logistic regression with forwards and backwards selection (AUC $=0.83)$, L1 (LASSO) penalization (AUC $=0.83$ ), L2 (Ridge) penalization (AUC $=0.83$ ), or combined L1 and L2 penalization $(\mathrm{AUC}=0.83)$ did not improve performance. Of 31 clinical actions in the base model, forwards and backwards selection retained 25 predictors. L1, L2, and combined L1-L2 penalization retained all 31 clinical 


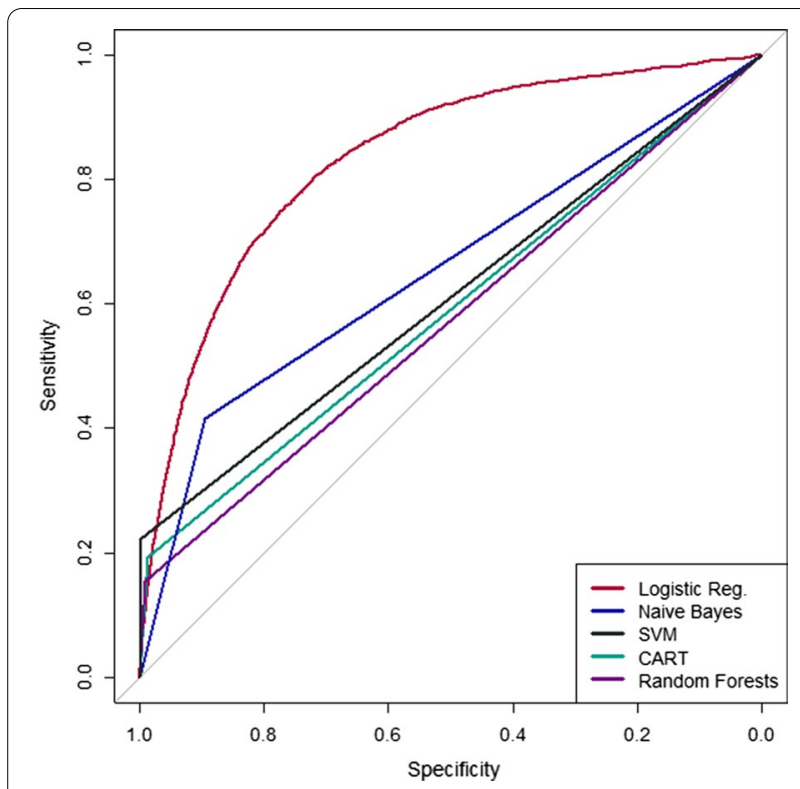

Fig. 1 Comparison of ROC curves for 5 binary classifiers for presence or absence of delirium in the critical care setting. By AUC, logistic regression (unadjusted odds) outperformed supervised binary classification by naïve Bayes, support vector machines (SVM), Classification and Regression Trees (CART), and random forests actions. Because three of four feature selection methods recommended inclusion of all 31 features and the potential for knowledge loss with predictor elimination, the model with 31 clinical actions was selected.

Table 3 presents 17 highly significant predictors $(\mathrm{p}<0.001)$ from the final, multiple logistic regression model of 31 clinical actions. The full model can be found in Additional file 2: Table A.3. Among clinical impressions captured from single words in text notes, odds of delirium were higher with each note mentioning "mental status" $(\mathrm{OR}=1.14)$, "deliri" $(\mathrm{OR}=1.12)$, "hallucin"" $(\mathrm{OR}=1.25)$, or "confus"” $(\mathrm{OR}=1.16)$, and "disorient" $(\mathrm{OR}=1.10)$. Odds of delirium were lower for each note mentioning "reorient" $(\mathrm{OR}=0.86)$. Among laboratory tests, odds of delirium were significantly greater with clinical orders for urine culture $(\mathrm{OR}=1.13)$, thyroid function test $(\mathrm{OR}=1.12)$, serum $\mathrm{B} 12$ or folate $(\mathrm{OR}=1.45)$, and blood or urine toxicology screen $(\mathrm{OR}=1.28)$. Prescription orders for antipsychotics $(\mathrm{OR}=1.44)$, benzodiazepines $(\mathrm{OR}=1.08)$, and dexmedetomidine $(\mathrm{OR}=1.43)$ were associated with higher odds of delirium.

\section{Reclassification and model comparison}

We compared six metrics for sensitivity (Se) tuning: the Youden Index $(\mathrm{Se}=80 \%)$, maximizing sensitivity and specificity $(\mathrm{Se}=80 \%)$, maximizing accuracy

Table 3 Highly significant predictors from a multiple logistic regression model to classify delirium in the medical record

\begin{tabular}{|c|c|c|c|c|c|}
\hline & Odds Ratio & $\begin{array}{l}95 \% \mathrm{Cl} \\
\text { Lower Bound }\end{array}$ & 95\% Cl Upper Bound & Z-Value & $p$-Value \\
\hline "Mental status" & 1.144 & 1.114 & 1.176 & 9.766 & $<0.000005$ \\
\hline "Deliri*" & 1.121 & 1.082 & 1.163 & 6.249 & $<0.000005$ \\
\hline "Hallucin*" & 1.252 & 1.161 & 1.351 & 5.820 & $<0.000005$ \\
\hline "Confus*" & 1.160 & 1.123 & 1.199 & 8.872 & $<0.000005$ \\
\hline "Reorient*" & 0.863 & 0.807 & 0.923 & -4.270 & 0.00002 \\
\hline Urine culture & 1.131 & 1.084 & 1.179 & 5.682 & $<0.000005$ \\
\hline $\mathrm{ABG}^{\mathrm{a}}$ & 0.978 & 0.972 & 0.984 & -7.097 & $<0.000005$ \\
\hline Renal function panel & 1.041 & 1.024 & 1.058 & 4.871 & $<0.000005$ \\
\hline$C B C^{b}$ & 0.965 & 0.952 & 0.978 & -5.186 & $<0.000005$ \\
\hline Thyroid function test & 1.122 & 1.058 & 1.189 & 3.872 & 0.00011 \\
\hline Toxicology screen & 1.275 & 1.217 & 1.336 & 10.243 & $<0.000005$ \\
\hline Autoimmune serology & 0.408 & 0.292 & 0.556 & -5.464 & $<0.000005$ \\
\hline B vitamins & 1.451 & 1.315 & 1.598 & 7.477 & $<0.000005$ \\
\hline HIV antibody & 0.479 & 0.314 & 0.705 & -3.574 & 0.00035 \\
\hline Antipsychotics & 1.443 & 1.400 & 1.488 & 23.589 & $<0.000005$ \\
\hline Benzodiazepines & 1.076 & 1.048 & 1.103 & 5.614 & $<0.000005$ \\
\hline Dexmedetomidine & 1.432 & 1.260 & 1.626 & 5.513 & $<0.000005$ \\
\hline
\end{tabular}

Presented here are 17 predictors from 31 clinical actions from a multiple logistic regression model with $p<0.001$. Coefficients and confidence intervals are presented for odds. The full model is available in Additional file 2: A.3

a Arterial blood gas

${ }^{b}$ Complete blood count 
(Se $=5.20 \%)$, minimizing the distance to $\operatorname{ROC}(0,1)$ ( $\mathrm{Se}=76 \%)$, maximizing accuracy constraining sensitivity $(\mathrm{Se}=50 \%)$, and maximizing sensitivity constraining specificity ( $\mathrm{Se}=92 \%$ ). Additional file 2: Table A.4 illustrates the cut-point, sensitivity, specificity, and accuracy of six methods for tuning a threshold for a binary logistic classifier. Figure 2 visualizes reclassification of the test cohort by our model into four groups (ICDPositives, Reclassified-Positives, Reclassified-Negatives, Double-Negatives) along our chosen method, the Youden Index. (Additional file 2: Figure A.1 presents this visualization for Puelle's classifier.)

On the test data, logistic regression with threshold reclassification by the Youden Index (cut-point $=-2.72$ ) and all 31 clinical predictors included resulted in a model with AUC of $0.83,79.4 \%$ sensitivity, $71.5 \%$ specificity, $19.7 \% \mathrm{PPV}$, and $97.6 \% \mathrm{NPV}$, evaluated against delirium ICD-9 codes. This threshold reclassification suggests a delirium prevalence within the ICU cohort up to $32.5 \%$. Puelle's classifier, using a Youden Index cut-point of -2.671 , produced $79.8 \%$ sensitivity, $72.2 \%$ specificity, $19.8 \% \mathrm{PPV}$, and $97.6 \% \mathrm{NPV}$, predicting a cohort delirium prevalence $31.9 \%$. Puelle's classifier had AIC of 18,378 and BIC of 18,455 . Our novel model had AIC of 16,786 and BIC of 17,058. By definition, Kim's reclassification categorized all ICD-Positives as having delirium and did not generate Reclassified-Negatives, resulting in $100 \%$ sensitivity, $85.7 \%$ specificity, $37.7 \%$ PPV, and $100 \%$ NPV, with an estimated cohort prevalence of delirium up to $21.1 \%$.

Table 4 illustrates similarity and difference between four reclassification groups on five clinical measures. With Kim's reclassifier, Double-Positives and Double-Negatives differed significantly for age, discharge location, death during hospitalization, and one-year mortality, but did not differ for 30-day mortality $(\mathrm{p}=0.472)$. Double-Positives and Reclassified-Negatives differed

Table 4 Significant differences for three models between four reclassification groups on five clinical measures

\begin{tabular}{|c|c|c|c|c|}
\hline & \multicolumn{4}{|c|}{ Reclassification Groups } \\
\hline & $\begin{array}{l}\text { Double+ } \\
\text { vs } \\
\text { Double- }\end{array}$ & $\begin{array}{l}\text { Double + } \\
\text { vs } \\
\text { Reclassified + }\end{array}$ & $\begin{array}{l}\text { Reclassified + } \\
\text { vs } \\
\text { Reclassified- }\end{array}$ & $\begin{array}{l}\text { Double- } \\
\text { vs } \\
\text { Reclassified- }\end{array}$ \\
\hline Expected Relationship & $\begin{array}{l}\text { Different } \\
p<0.05^{a}\end{array}$ & $\begin{array}{l}\text { Same } \\
p>0.05^{a}\end{array}$ & $\begin{array}{l}\text { Different } \\
p<0.05^{a}\end{array}$ & $\begin{array}{l}\text { Same } \\
p>0.05^{a}\end{array}$ \\
\hline \multicolumn{5}{|l|}{ Novel Model } \\
\hline Age & $<0.00005$ & $<0.00005$ & $<0.00005$ & $<0.00005$ \\
\hline Discharge location & $<0.00005$ & $<0.00005$ & $<0.00005$ & $<0.00005$ \\
\hline Death in hospital & $<0.00005$ & 0.00011 & 0.0452 & 0.0859 \\
\hline 30-day mortality & 0.00072 & 0.115 & 0.00017 & 0.0011 \\
\hline one-year mortality & $<0.00005$ & 0.178 & 0.0017 & $<0.00005$ \\
\hline \multicolumn{5}{|l|}{ Puelle's Classifier } \\
\hline Age & $<0.00005$ & 0.00097 & $<0.00005$ & 0.964 \\
\hline Discharge location & $<0.00005$ & $<0.00005$ & 0.00062 & 0.00062 \\
\hline Death in hospital & 0.0017 & $<0.00005$ & 0.820 & 0.103 \\
\hline 30-day mortality ${ }^{b}$ & 0.0949 & - & - & - \\
\hline one-year mortality & $<0.00005$ & $<0.00005$ & 0.660 & 0.0015 \\
\hline \multicolumn{5}{|l|}{ Kim's Classifier } \\
\hline Age & $<0.00005$ & 0.0130 & - & - \\
\hline Discharge location & $<0.00005$ & $<0.00005$ & - & - \\
\hline Death in hospital & 0.0035 & $<0.00005$ & - & - \\
\hline 30-day mortality ${ }^{b}$ & 0.472 & - & - & - \\
\hline one-year mortality & $<0.00005$ & 0.0010 & - & - \\
\hline
\end{tabular}

Binary classification generates four groups of subjects, including two groups of reclassified hospitalizations, which were compared by biological validation to assess model goodness-of-fit. Double-Positives are expected to differ from Double-Negatives. In the case of successful reclassification, we expect Double-Positives to be similar to Reclassified-Positives, Double-Negatives to be similar to Reclassified-Negatives, and Reclassified-Positives to differ from Reclassified-Negatives. Bolded fields represent $\mathrm{p}$-values concordant with expectations of good model fit

${ }^{a}$ p-values were generated from pairwise chi-squared testing with the Holm correction for all measures except age, which was tested with pairwise Tukey multiple comparisons of means

b Pairwise testing was not performed in the event of no significant difference between Double-Positives and Double-Negatives

c The Kim model does not generate reclassified negatives, making associated tests unavailable 


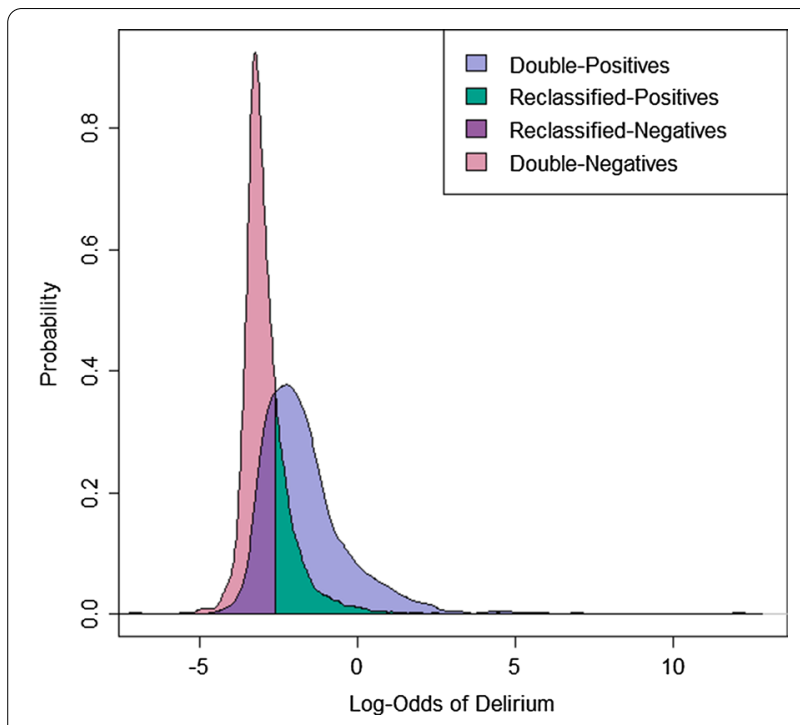

Fig. 2 Probability density plot of four reclassification groups generated by our model predicting delirium from 31 clinical actions. Binary classification by multiple logistic regression generates four groups, including two groups of reclassified hospitalizations

significantly in all tested categories. Under Puelle's classifier, Double-Positives and ICD-Negatives differed significantly in all clinical validation measures except 30-day mortality $(\mathrm{p}=0.949)$. Reclassified-Negatives and DoubleNegatives did not significantly differ for age $(p=0.964)$ and death in hospital $(\mathrm{p}=0.103)$. However, ICD-Positive and Reclassified-Positives differed significantly on all tested validation measures. Reclassified-Positives and Reclassified-Negatives did not differ significantly by death in hospital $(\mathrm{p}=0.820)$ or one-year mortality (0.660). In our novel model, Double-Positives and Double-Negatives differed significantly on all five validation measures. Double-Positives and Reclassified-Positives did not significantly differ by 30 -day mortality $(\mathrm{p}=0.115)$ or one-year mortality $(\mathrm{p}=0.178)$. Double-Negatives and Reclassified-Negatives did not differ significantly by death in hospital. Reclassified-Positives and ReclassifiedNegatives differed significantly $(\mathrm{p}<0.05)$ for all 5 validation measures.

\section{Discussion}

ML holds the potential to unlock improved diagnosis, risk stratification, and treatment of delirium in the ICU, a complex syndrome associated with serious morbidity and mortality. Before ML can be used to make clinically actionable predictions, informaticians developing models for delirium incidence, prognosis, and treatment need tools to accurately label patients with delirium in large datasets, despite serious flaws with current labeling methods. Ideally, delirium researchers need a valid, efficient, computational tool that is independent of clinical variable of interest to label patients with delirium in large datasets without the need for chart review on in-person clinical assessments. A high-accuracy, computationally-generated label could be used for training future models on pressing clinical questions, including identifying timing of delirium onset in the hospital course or classifying patients with delirium into clinically relevant clusters. Here, we proposed to label delirium from clinician actions, using placement of orders associated with standard workup of delirium as a surrogate for clinicians recognizing delirium in real time.

After comparison of five supervised ML methods and four methods of feature selection, we proposed a novel, multiple logistic regression model to label ICU delirium from counts of 31 clinician actions abstracted from clinical guidelines, with high AUC (0.83). If predictors are not independent, we expect improved performance from non-linear models. However, because these 31 clinical actions are regularly employed in wider clinical practice independent of delirium and thus none are specific for delirium, it is possible that a greater than expected independence between covariates resulted in unexpectedly good performance from the logistic model. The assumption of independence is reinforced by a correlation matrix with less than $4 \%$ of 31 predictors having a Spearman's $\rho$ of $\geq 0.6$. The logistic model is both appropriate to the data and offers clearer, biological interpretability than many non-linear models.

Model performance on a training set was validated on a randomly selected test set. The model was concordant with clinical intuition, with odds of delirium higher with words such as "deliri"," "hallucin", and "disorient", but odds of delirium lower with "reorient"." Marked elevations in odds of delirium were associated with toxicology screening, used to detect delirium from substance intoxication or withdrawal, and prescription of antipsychotics or dexmedetomidine. Evidence of intoxication falls within the DSM-5 criteria for diagnosis of delirium $[1,12]$. Guidelines recommend antipsychotics as the drug class of choice for symptomatic treatment of delirium [13]. Dexmedetomidine is recommended as a preferred drug for management of delirium on mechanically ventilated patients [13].

We compared our labeling model to two similar models previously proposed in the literature to abstract delirium incidence from chart review. Both our model and Puelle's classifier produced sensitivity and specificity between 71 and $80 \%$, indicating good fidelity to delirium ICD-9 codes with modest reclassification of both positives and negatives. Although the implementation of Puelle's classifier has similar PPV and sensitivity with fewer predictors, 
our novel model had superior performance by both lower $\mathrm{AIC}$ and BIC.

Kim et al. [24] reported low sensitivity (30\%) but high specificity (97\%) of their classifier on a prospective study of 184 adults. Specificity on the expanded MIMIC-III data set was $85.7 \%$. Our implementation of Kim classifier never generates reclassified negatives: all patients with ICD-9 codes for delirium are classified in the delirium group by definition. Thus, the $100 \%$ sensitivity and $100 \%$ NPV reflect definitions for model creation, not quality of fit. The PPV of Kim's classifier (37.7\%) surpasses that of Puelle's classifier (19.8\%) and our model (19.7\%). However, PPV is also defined by simple re-categorization in Kim's classifier, and is not indicative of improved performance. For both Kim's and Puelle's classifiers, reduced performance with computational application on the expanded, MIMIC-III dataset suggest limitations in generalizability and validation of these small-scale proposals.

Because ground-truth is not reasonably attainable in these data by chart review due to their very large size, we compared goodness-of-fit of the three models by biological validation [26]. First, we assume that, for a good model, predicted prevalence of delirium (sum of ICDPositives and Reclassified-Positives) should approach known ICU delirium prevalence from the literature. In a meta-analysis of 48 studies on ICU delirium, Krewulak et al. [2] obtained an overall pooled delirium prevalence of $31 \%$. Kim's classifier predicted delirium prevalence above ICD-9 code frequency (21.1\%). Our model (32.5\%) and Puelle's classifier (31.9\%) predicted delirium prevalence concordant with Krewulak's pooled figures, indicating an appropriate quantity of reclassified patients.

We further biologically validate against clinically meaningful outcome measures. We compared classification and reclassification groups by age, discharge location, short-term risk of death, and one-year mortality. Our method of model validation rests on the principle that application of any binary classifier that does not have perfect $(100 \%)$ sensitivity and specificity reclassifies subjects, such that some number of subjects receive a classification from the model that differs from their input label assignment (Table 1, Fig. 2). If the binary classification model is valid, then this unavoidable reclassification should result in reclassified subjects resembling their reclassified assignment more so than their label assignment across the five comparison measures. On the basis of biological validation, our novel model markedly outperformed Kim's and Puelle's classifiers, correctly capturing significant differences between Double-Positives and Double-Negatives and between Reclassified-Positives and Reclassified-Negatives on all five measures. Delirium is a heterogeneous syndrome with subtype variation, including an underdiagnosed hypoactive subtype and a subclinical form $[5,12]$. Thus, differences between Double-Positives and Reclassified-Positives may represent variability in clinician practice between delirium subtypes, with our model reclassifying patients belonging to subtypes underrepresented in previous studies.

\section{Limitations}

The clinical utility of our novel model rests on important contextual factors. First, our study is based on publicly available data from one institution. However, our model uses one of the largest count of observations for developing a ML model for delirium than previously used in other studies. Although we propose the implementation of a generalizable labeling model that is relatively less labor intensive than models that depend upon screening tools, ICD codes, and chart review (many of which that are not easily available), we recognize the importance of heterogeneity that will exist at both an institutional and a local provider level [62]. Examples include sub-group and temporal considerations and idiosyncratic coding and documentation practices. There is a need for local validation and recalibration to ensure the optimal performance of our labeling method [63]. Because of under-identification of hypoactive or milder delirium in the clinical[5] or analytic[24] setting, deviations in model goodness of fit may reflect variation in clinical practice and patient presentation between delirium subtypes.

As noted previously, our model's overall performance, albeit relatively better than other counterpart models, still has constraints in terms of factors such as sensitivity and PPV. Like other ML models, decisions to implement our model will require considerations about tradeoffs around model performance factors, the costs of model implementation, and the implications of falsepositives $[64,65]$. The potential response to positive cases and other approaches that can be used to establish true-positive cases will be critical. Finally, because this model does not use time-dependent variables, it may not be able to label a patient with delirium until after all encounter data is available.

Future work to predict delirium subtypes from the medical record is warranted. Patients being presented with other diseases, example SARS-CoV-2, may result in the introduction of other features that may improve the calibration of the model given the prevalence of such a disease in the local ICU. ICU delirium has been shown to be comorbid with SARS-CoV-2, arising from disorientation and social isolation, use of mechanical ventilation, and an aging patient population [66]. 


\section{Conclusions}

We developed a novel labeling model for delirium in the ICU using a large data set from a publicly available database. This database has been previously used to develop ML models for other applications [67, 68]. Our model incorporates 31 clinical actions as features, an approach that has been previously overlooked in other delirium prediction models. We assessed the performance of our labeling model based on other delirium prediction models and biological markers of significance. Our model demonstrates relative superiority based on the assessment rubric; however, more validation and recalibration are needed to consider important contextual factors that may arise before and during the use of the model in a local ICU. These results provide a tool to aid future researchers developing ML classifiers for ICU patients with delirium.

\begin{abstract}
Abbreviations
AIC: Akaike information criterion; AUC: Area under the receiver operating characteristic curve; BIC: Bayes information criterion; CAM-ICU: Confusion assessment method; CART: Classification and regression trees; DSM: Diagnostic and statistical manual of mental disorders, 5th Edition; EHR: Electronic health record; ICU: Intensive care unit; ICD: International classification of diseases; ICDSC: Intensive care delirium screening checklist; LASSO: Least absolute shrinkage and selection operator; LOS: Length of stay; ML: Machine learning; MIMIC: Medical information mart for intensive care; PPV: Positive predictive value; PU: Positive unlabeled; SARS-CoV-2: Severe acute respiratory syndrome coronavirus 2; TRIPOD: Transparent reporting of a multivariable prediction model for individual prognosis or diagnosis; NPV: Negative predictive value; OR: Odds ratio.
\end{abstract}

\section{Supplementary Information}

The online version contains supplementary material available at https://doi, org/10.1186/s12911-021-01461-6.

Additional file 1. Study model development and model comparison tables.

Additional file 2. Study R Markdown file with data pre-processing and variable selection.

\section{Acknowledgements}

The authors do not have any personal acknowledgements.

\section{Authors' contributions}

CEC contributed in study design, data analysis, and manuscript preparation. KRC contributed in data analysis and manuscript preparation. NF contributed in study design, data analysis, and manuscript preparation. All authors read and approved the final manuscript.

\section{Funding}

The authors do not have any outside funding for this project to acknowledge.

\section{Availability of data and materials}

The dataset supporting the conclusions of this article is available in the MIMICIII repository,[38, 39, 41] https://mimic.physionet.org/.

\section{Declarations}

\section{Ethics approval and consent to participate}

Permission to use the dataset supporting our work was granted by PhysioNet. Only PhysioNet credentialed users who sign a data use agreement can access the Medical Information Mart for Intensive Care-III (MIMIC-III) repository files.

\section{Consent for publication}

Not applicable.

\section{Competing interests}

The authors have no competing interests to declare.

\section{Author details}

${ }^{1}$ College of Medicine, The Ohio State University, Columbus, OH 43210, USA. ${ }^{2}$ Department of Biomedical Informatics, The Ohio State University College of Medicine, 460 Medical Center Dr., 512 Institute of Behavioral Medicine Research, Columbus, OH 43210, USA. ${ }^{3}$ Center for the Advancement of Team Science, Analytics, and Systems Thinking, College of Medicine, The Ohio State University, Columbus, $\mathrm{OH}$ 43210, USA.

Received: 2 September 2020 Accepted: 2 March 2021

Published online: 09 March 2021

\section{References}

1. Association AP: Diagnostic and statistical manual of mental disorders (DSM-5 ${ }^{\circledR}$ ): American Psychiatric Pub; 2013

2. Krewulak KD, Stelfox HT, Leigh JP, Ely EW, Fiest KM. Incidence and prevalence of delirium subtypes in an adult ICU: a systematic review and meta-analysis. Crit Care Med. 2018;46(12):2029-35.

3. Inouye SK, Westendorp RG, Saczynski JS. Delirium in elderly people. Lancet (London, England). 2014;383(9920):911-22.

4. Ely EW, Shintani A, Truman B, Speroff T, Gordon SM, Harrell FE Jr, Inouye SK, Bernard GR, Dittus RS. Delirium as a predictor of mortality in mechanically ventilated patients in the intensive care unit. JAMA. 2004;291(14):1753-62.

5. Marcantonio ER. Delirium in hospitalized older adults. N Engl J Med. 2017;377(15):1456-66.

6. Marcantonio ER: In the clinic. Delirium. Annals Internal Med 2011, 154(11):ITC6-1, ITC6-2, ITC6-3, ITC6-4, ITC6-5, ITC6-6, ITC6-7, ITC6-8, ITC6-9, ITC6-10, ITC16-11, ITC16-12, ITC16-13, ITC16-14, ITC16-15; quiz ITC16-16.

7. Gleason LJ, Schmitt EM, Kosar CM, Tabloski P, Saczynski JS, Robinson T, Cooper Z, Rogers SO Jr, Jones RN, Marcantonio ER, et al. Effect of delirium and other major complications on outcomes after elective surgery in older adults. JAMA Surg. 2015;150(12):1134-40.

8. Saczynski JS, Marcantonio ER, Quach L, Fong TG, Gross A, Inouye SK, Jones RN. Cognitive trajectories after postoperative delirium. N Engl J Med. 2012;367(1):30-9.

9. Pandharipande PP, Girard TD, Jackson JC, Morandi A, Thompson JL, Pun BT, Brummel NE, Hughes CG, Vasilevskis EE, Shintani AK, et al. Long-term cognitive impairment after critical illness. N Engl J Med. 2013;369(14):1306-16.

10. Witlox J, Eurelings LS, de Jonghe JF, Kalisvaart KJ, Eikelenboom P, van Gool WA. Delirium in elderly patients and the risk of postdischarge mortality, institutionalization, and dementia: a meta-analysis. JAMA 2010;304(4):443-51.

11. Fong TG, Tulebaev SR, Inouye SK. Delirium in elderly adults: diagnosis, prevention and treatment. Nat Rev Neurol. 2009;5(4):210-20.

12. Lawlor PG, Bush SH. Delirium diagnosis, screening and management. Curr Opin Support Palliat Care. 2014;8(3):286-95.

13. Barr J, Fraser GL, Puntillo K, Ely EW, Gelinas C, Dasta JF, Davidson JE, Devlin JW, Kress JP, Joffe AM, et al. Clinical practice guidelines for the management of pain, agitation, and delirium in adult patients in the intensive care unit. Crit Care Med. 2013:41(1):263-306.

14. Ciampi A, Bai C, Dyachenko A, McCusker J, Cole MG, Belzile E. Latent class analysis of the multivariate Delirium Index in long-term care settings. Int Psychogeriatr. 2019;31(1):59-72. 
15. Lagarto L, Cerejeira J. Identification of sub-groups in acutely ill elderly patients with delirium: a cluster analysis. Int Psychogeriatr. 2016;28(8):1283-92.

16. Wong A, Young AT, Liang AS, Gonzales R, Douglas VC, Hadley D. Development and validation of an electronic health record-based machine learning model to estimate delirium risk in newly hospitalized patients without known cognitive impairment. JAMA Netw Open. 2018;1(4):e181018.

17. Oh J, Cho D, Park J, Na SH, Kim J, Heo J, Shin CS, Kim JJ, Park JY, Lee B. Prediction and early detection of delirium in the intensive care unit by using heart rate variability and machine learning. Physiol Meas. 2018;39(3):035004.

18. Corradi JP, Thompson S, Mather JF, Waszynski CM, Dicks RS. Prediction of incident delirium using a random forest classifier. J Med Syst. 2018;42(12):261.

19. Mufti HN, Hirsch GM, Abidi SR, Abidi SSR. Exploiting machine learning algorithms and methods for the prediction of agitated delirium after cardiac surgery: models development and validation study. JMIR Med Inf. 2019;7(4):e14993.

20. Halladay CW, Sillner AY, Rudolph JL. Performance of electronic prediction rules for prevalent delirium at hospital admission. JAMA Netw Open. 2018;1(4):e181405.

21. Rudolph JL, Doherty K, Kelly B, Driver JA, Archambault E. Validation of a delirium risk assessment using electronic medical record information. J Am Med Dir Assoc. 2016;17(3):244-8.

22. Davoudi A, Ebadi A, Rashidi P, Ozrazgat-Baslanti T, Bihorac A, Bursian AC. Delirium prediction using machine learning models on preoperative electronic health records data. Proc IEEE Int Symp Bioinf Bioeng. 2017;2017:568-73.

23. Ely EW, Inouye SK, Bernard GR, Gordon S, Francis J, May L, Truman B, Speroff T, Gautam S, Margolin R, et al. Delirium in mechanically ventilated patients: validity and reliability of the confusion assessment method for the intensive care unit (CAM-ICU). JAMA. 2001;286(21):2703-10.

24. Kim DH, Lee J, Kim CA, Huybrechts KF, Bateman BT, Patorno E, Marcantonio ER. Evaluation of algorithms to identify delirium in administrative claims and drug utilization database. Pharmacoepidemiol Drug Saf. 2017:26(8):945-53.

25. Puelle MR, Kosar CM, Xu G, Schmitt E, Jones RN, Marcantonio ER, Cooper Z, Inouye SK, Saczynski JS. The language of delirium: keywords for identifying delirium from medical records. J Gerontol Nurs. 2015;41(8):34-42.

26. Coombes CE, Abrams ZB, Li S, Abruzzo LV, Coombes KR: Unsupervised machine learning and prognostic factors of survival in chronic lymphocytic leukemia. JAMIA 2020.

27. Veeranki S, Hayn D, Eggerth A, Jauk S, Kramer D, Leodolter W, Schreier G. On the representation of machine learning results for delirium prediction in a hospital information system in routine care. Stud Health Technol Inf. 2018:251:97-100

28. Devlin JW, Fong JJ, Schumaker G, O'Connor H, Ruthazer R, Garpestad E: Use of a validated delirium assessment tool improves the ability of physicians to identify delirium in medical intensive care unit patients. Critical Care Med 2007, 35(12):2721-2724; quiz 2725.

29. Bergeron N, Dubois MJ, Dumont M, Dial S, Skrobik Y. Intensive care delirium screening checklist: evaluation of a new screening tool. Intensive Care Med. 2001;27(5):859-64.

30. O'Malley KJ, Cook KF, Price MD, Wildes KR, Hurdle JF, Ashton CM. Measuring diagnoses: ICD code accuracy. Health Serv Res. 2005;40(5 Pt 2):1620-39

31. Wells BJ, Chagin KM, Nowacki AS, Kattan MW. Strategies for handling missing data in electronic health record derived data. EGEMS (Washington, DC). 2013;1(3):1035.

32. Horsky J, Drucker EA, Ramelson HZ. Accuracy and completeness of clinical coding using ICD-10 for ambulatory visits. AMIA Ann Symp Proc AMIA Symp. 2017;2017:912-20.

33. Rothman KJ, Greenland S, Lash TL: Modern epidemiology: Lippincott Williams \& Wilkins; 2008

34. Reibnegger G, Schrabmair W. Optimum binary cut-off threshold of a diagnostic test: comparison of different methods using Monte Carlo technique. BMC Med Inform Decis Mak. 2014;14:99.

35. Gilbert R, Martin RM, Donovan J, Lane JA, Hamdy F, Neal DE, Metcalfe C. Misclassification of outcome in case-control studies: Methods for sensitivity analysis. Stat Methods Med Res. 2016;25(5):2377-93.
36. Bekker J, Davis J: Learning from positive and unlabeled data: A survey. arXiv preprint arXiv:1811.04820;2018.

37. Harrell FE, Jr., Margolis PA, Gove S, Mason KE, Mulholland EK, Lehmann D, Muhe L, Gatchalian S, Eichenwald HF: Development of a clinical prediction model for an ordinal outcome: the World Health Organization Multicentre Study of Clinical Signs and Etiological agents of Pneumonia, Sepsis and Meningitis in Young Infants. WHO/ARI Young Infant Multicentre Study Group. Stat Med 1998, 17(8):909-944.

38. Johnson AEW, Pollard TJ, Shen L. Lehman L-wH, Feng M, Ghassemi M, Moody B, Szolovits P, Anthony Celi L, Mark RG: MIMIC-III, a freely accessible critical care database. Scientific Data. 2016;3(1):160035.

39. Johnson AE, Stone DJ, Celi LA, Pollard TJ. The MIMIC Code Repository: enabling reproducibility in critical care research. J Am Med Inform Assoc. 2018;25(1):32-9.

40. Goldberger AL, Amaral LA, Glass L, Hausdorff JM, Ivanov PC, Mark RG, Mietus JE, Moody GB, Peng C-K, Stanley HE: PhysioBank, PhysioToolkit, and PhysioNet: components of a new research resource for complex physiologic signals. Circulation 2000, 101(23):e215-e220.

41. Pollard TJaJ, Alistair EW: The MIMIC-III Clinical Database; 2016.

42. Jameson JL: Harrison's principles of internal medicine: McGraw-Hill Education; 2018.

43. Fohner AE, Greene JD, Lawson BL, Chen JH, Kipnis P, Escobar GJ, Liu VX. Assessing clinical heterogeneity in sepsis through treatment patterns and machine learning. JAMIA. 2019;26(12):1466-77.

44. Breiman L, Friedman J, Stone CJ, Olshen RA: Classification and regression trees: CRC press; 1984.

45. Therneau TM, Atkinson EJ. An introduction to recursive partitioning using the RPART routines. In.: Technical report Mayo Foundation; 1997.

46. Breiman L. Random forests. Mach Learn. 2001;45(1):5-32.

47. Liaw A, Wiener M. Classification and regression by randomForest. R news. 2002;2(3):18-22.

48. Maron ME. Automatic indexing: an experimental inquiry. JACM. 1961;8(3):404-17.

49. Dimitriadou E, Hornik K, Leisch F, Meyer D, Weingessel A, Leisch MF: The e1071 package. Misc Functions of Department of Statistics (e1071), TU Wien 2006

50. Cortes C, Vapnik V. Support-vector networks. Machine learning. 1995;20(3):273-97.

51. Santosa F, Symes WW. Linear inversion of band-limited reflection seismograms. SIAM J Sci Stat Comput. 1986;7(4):1307-30.

52. Tibshirani R. Regression shrinkage and selection via the lasso. J Roy Stat Soc: Ser B (Methodol). 1996;58(1):267-88.

53. Hoerl AE, Kennard RW. Ridge regression: Biased estimation for nonorthogonal problems. Technometrics. 1970;12(1):55-67.

54. Goeman J, Meijer R, Chaturvedi N: L1 and L2 penalized regression models. Vignette R Package Penalized; 2018. https://cran.r-project.org/web/ packages/penalized/vignettes/penalized.pdf.

55. Robin X, Turck N, Hainard A, Tiberti N, Lisacek F, Sanchez J-C, Müller M. pROC: an open-source package for $\mathrm{R}$ and $\mathrm{S}+$ to analyze and compare ROC curves. BMC Bioinformatics. 2011;12(1):1-8.

56. Collins GS, Reitsma JB, Altman DG, Moons KG. Transparent Reporting of a multivariable prediction model for Individual Prognosis or Diagnosis (TRIPOD): the TRIPOD statement. Ann Intern Med. 2015;162(1):55-63.

57. Youden WJ. Index for rating diagnostic tests. Cancer. 1950;3(1):32-5.

58. Thiele C, Hirschfeld G: The cutpointr package: Improved and tidy estimation of optimal cutpoints. Ulmer Informatik-Berichte 2017:14.

59. Dziak JJ, Coffman DL, Lanza ST, Li R, Jermiin LS. Sensitivity and specificity of information criteria. Brief Bioinform. 2020 Mar 23;21(2):553-65.

60. Shan G, Gerstenberger S. Fisher's exact approach for post hoc analysis of a chi-squared test. PLoS ONE. 2017;12(12):e0188709.

61. Holm S: A simple sequentially rejective multiple test procedure. Scand J Stat;1979:65-70.

62. Reps JM, Schuemie MJ, Suchard MA, Ryan PB, Rijnbeek PR. Design and implementation of a standardized framework to generate and evaluate patient-level prediction models using observational healthcare data. JAMIA. 2018;25(8):969-75.

63. Ding X, Gellad ZF, Mather C 3rd, Barth P, Poon EG, Newman M, Goldstein BA. Designing risk prediction models for ambulatory no-shows across different specialties and clinics. JAMIA. 2018;25(8):924-30. 
64. Jehi L, Ji X, Milinovich A, Erzurum S, Rubin B, Gordon S, Young J, Kattan MW: Individualizing risk prediction for positive COVID-19 testing: results from 11,672 patients. Chest 2020.

65. Grundmeier RW, Xiao R, Ross RK, Ramos MJ, Karavite DJ, Michel JJ, Gerber JS, Coffin SE. Identifying surgical site infections in electronic health data using predictive models. JAMIA. 2018;25(9):1160-6.

66. Kotfis K, Williams Roberson S, Wilson JE, Dabrowski W, Pun BT, Ely EW. COVID-19: ICU delirium management during SARS-CoV-2 pandemic. Critical Care (London, England). 2020;24(1):176.

67. Huang J, Osorio C, Sy LW. An empirical evaluation of deep learning for ICD-9 code assignment using MIMIC-III clinical notes. Comput Methods Prog Biomed. 2019;177:141-53.
68. Harutyunyan H, Khachatrian H, Kale DC, Ver Steeg G, Galstyan A. Multitask learning and benchmarking with clinical time series data. Sci Data. 2019;6(1):96.

\section{Publisher's Note}

Springer Nature remains neutral with regard to jurisdictional claims in published maps and institutional affiliations.
Ready to submit your research? Choose BMC and benefit from:

- fast, convenient online submission

- thorough peer review by experienced researchers in your field

- rapid publication on acceptance

- support for research data, including large and complex data types

- gold Open Access which fosters wider collaboration and increased citations

- maximum visibility for your research: over 100M website views per year

At BMC, research is always in progress.

Learn more biomedcentral.com/submissions 